\title{
Early Neural Correlates of Conscious Somatosensory Perception
}

\author{
Satu Palva, ${ }^{1,2}$ Klaus Linkenkaer-Hansen, ${ }^{3}$ Risto Näätänen, ${ }^{1,2}$ and J. Matias Palva ${ }^{4}$ \\ ${ }^{1}$ BioMag Laboratory, Engineering Centre, Helsinki University Central Hospital, FIN-00029 Helsinki, Finland, ${ }^{2}$ Cognitive Brain Research Unit, Department \\ of Psychology, University of Helsinki and Helsinki Brain Research Centre, FIN-00014 Helsinki, Finland, ${ }^{3}$ Netherlands Institute for Brain Research, 1105 \\ Amsterdam, The Netherlands, and ${ }^{4}$ Department of Biological and Environmental Sciences, University of Helsinki, FIN-00014 Helsinki, Finland
}

The cortical processing of consciously perceived and unperceived somatosensory stimuli is thought to be identical during the first $100-120 \mathrm{~ms}$ after stimulus onset. Thereafter, the electrophysiological correlates of conscious perception have been shown to be reflected in the $\mathrm{N} 1$ component of the evoked response as well as in later $(>200 \mathrm{~ms})$ nonstimulus-locked $\gamma$-band $(28-50 \mathrm{~Hz})$ oscillatory activity. To evaluate more specifically the time course and correlation of neuronal oscillations with conscious perception, we recorded neuromagnetic responses to threshold-intensity somatosensory stimuli. We show here that cortical broadband activities phase locked to the subsequently perceived stimuli in somatosensory, frontal, and parietal regions as early as 30-70 ms from stimulus onset, whereas the phase locking to the unperceived stimuli was weak and primarily restricted to somatosensory regions. Such stimulus locking also preceded the perceived stimuli, indicating that the phase of ongoing cortical activities biases subsequent perception. Furthermore, the data show that the stimulus locking was present in the $\theta-(4-8 \mathrm{~Hz}), \alpha-(8-14 \mathrm{~Hz}), \beta-(14-28 \mathrm{~Hz})$, and $\gamma-(28-40 \mathrm{~Hz})$ frequency bands, of which the widespread $\alpha$-band component was dominant for the consciously perceived stimuli but virtually unobservable for the unperceived stimuli. Our results show that the neural correlates of conscious perception are already found during the earliest stages of cortical processing from 30 to $150 \mathrm{~ms}$ after stimulus onset and suggest that $\alpha$-frequency-band oscillations have a role in the neural mechanisms of sensory awareness.

Key words: $\alpha$ oscillations; consciousness; evoked; phase locking; network; sensorimotor; somatosensory; synchrony; temporal; topography

\section{Introduction}

The patterns of event-related neuronal activity leading to a conscious perception have remained enigmatic. Early cortical responses (less than $\sim 100$ ms from stimulus onset) evoked by somatosensory stimuli have been found to be similar for consciously perceived and unperceived stimuli (Libet et al., 1967; Ray et al., 1999; Meador et al., 2002). The earliest component, N1, of the auditory (Parasuraman and Beatty, 1980) and visual (Supèr et al., 2001; Pins and Ffytche, 2003) evoked responses (ERs) thus far found to reflect stimulus detection peaks at $\sim 100-120 \mathrm{~ms}$ after stimulus onset. Electrophysiological and functional imaging studies have shown that not only the processing of the consciously perceived stimuli (Sahraie et al., 1997; Feinstein et al., 2004; Marois et al., 2004) but also the processing of imperceptible sensory stimuli (Libet et al., 1967; Sahraie et al., 1997; Colder and Tanenbaum, 1999; Ray et al., 1999; Meador et al., 2002; Blankenburg et al., 2003) involves a widespread network, including the primary sensory cortex (Libet et al., 1967; Sahraie et al., 1997;

Received Sept. 4, 2004; revised April 13, 2005; accepted April 18, 2005.

This work was supported by the Danish Research Agency, the Academy of Finland, the Sigrid Juselius Foundation, the Ella and Georg Ehrnrooth Foundation, the Foundation for the Advancement of Technology, the Oskar Öflund Foundation, and the Women's Science Foundation. We thank Kristian Donner, Riitta Hari, and Kai Kaila for helpful discussions and critical comments on previous versions of this manuscript and V. V. Nikulin for technical assistance.

Correspondence should be addressed to Satu Palva, BioMag Laboratory, Engineering Centre, Helsinki University Central Hospital, P.0. Box 340, FIN-00029 Helsinki, Finland. E-mail: satu.palva@helsinki.fi.

DOI:10.1523/JNEUROSCI.0141-05.2005

Copyright $\odot 2005$ Society for Neuroscience $\quad$ 0270-6474/05/255248-11\$15.00/0
Colder and Tanenbaum, 1999; Ray et al., 1999; Meador et al., 2002; Blankenburg et al., 2003), as well as several areas higher in the processing hierarchy (Brazdil et al., 2001; Naccache and Dehaene, 2001; Blankenburg et al., 2003). Together, these data suggest that the neural correlates of conscious perception emerge at later stages and higher levels of sensory processing, after an initial period of 100-120 ms, during which stimulus-locked neuronal processing does not distinguish the subsequently perceived stimuli from those escaping conscious perception.

Phase synchronization of neuronal activity has been hypothesized to underlie the emergence of dynamic neuronal assemblies that constitute the metarepresentations required by sensory awareness (Singer, 2002). Indeed, $\gamma$-frequency-band phase synchrony in the primary somatosensory cortex is enhanced at $\sim 200$ ms after the onsets of consciously perceived somatosensory stimuli (Meador et al., 2002). Moreover, studies on binocular rivalry have shown that strengthened local (Fries et al., 1997) and largescale (Tononi et al., 1998; Srinivasan et al., 1999) neuronal synchrony is correlated with conscious stimulus perception, hence emphasizing the role of neuronal phase relationships in sensory awareness.

We investigated the neural correlates of sensory perception by examining the event-related phase and amplitude dynamics of neural network activities recorded using magnetoencephalography (MEG). We show here that these correlates emerge as early as $30 \mathrm{~ms}$ after the onset of somatosensory stimuli and that the large- 
scale stimulus locking in the $\alpha$-frequency band is selectively correlated with conscious perception.

\section{Materials and Methods}

Subjects and recordings. Cortical activity was recorded from 14 subjects (23-32 years of age; one female) with a 306 channel MEG instrument (Elekta Neuromag, Helsinki, Finland); the signals from the 204 planar gradiometers of the device were used in this study. Electro-oculograms and thumb-movement electromyograms were recorded for artifact rejection and for the detection of subjects' responses (see below), respectively. The study was approved by an ethical committee of the Helsinki University Central Hospital.

Experimental protocol. Subjects were electrically stimulated on the tip of the index finger with weak $0.2 \mathrm{~ms}$ constant-current pulses. The stimuli were applied to the left, right, or simultaneously to both index fingers equiprobably in a semirandom order. The interval between the consecutive pulses (stimulus onset asynchrony) varied randomly from 3 to $4 \mathrm{~s}$. Four 20 min sessions were recorded for each subject. Before each recording session, the intensity was separately adjusted for both fingers, so that maximally $50 \%$ of the trials were detected (for behavioral data, see Results and Fig. 1). During the recording sessions, subjects sat relaxed with their eyes closed and were instructed to promptly twitch the thumb of the hand(s) in which they had felt the stimulus. The thumb twitches were detected off-line from electromyographic (EMG) recordings that were bandpass filtered from 50 to $150 \mathrm{~Hz}$. The latency at which the EMG burst exceeded 10 baseline SDs was defined as the reaction time.

For all analyses of MEG data (see below), the stimuli, followed by correct responses, were categorized as consciously perceived (e.g., a right thumb twitch after a right index finger stimulus). Stimuli to which no response was observed were categorized as unperceived. Other stimulusresponse combinations (i.e., wrong responses) were not analyzed because of the poor signal-to-noise ratio in this category (for their prevalence, see Fig. 1). All responses occurring from 0.1 to $3 \mathrm{~s}$ after the stimulus onset were accepted.

The responses to left- and right-hand stimuli were pooled so that data in gradiometer selections with hemispheric asymmetry [i.e., the channel selections for sensorimotor (SM), primary somatosensory cortex (SI), and secondary somatosensory cortex (SII)] (see Fig. 2) were averaged within contralateral and ipsilateral categories (for instance, in Fig. 4, contralateral SI was obtained by averaging the responses to a left-finger stimulus over right hemispheric SI with the responses to a right-finger stimulus over the left hemispheric SI). The topographic visualizations (see Figs. $2 B, 3 C, 5 B$ ) were shown for right-finger stimuli only. The topographic maps for left-finger stimuli were very similar, except for the opposite hemispheric lateralization. The MEG data on event-related responses to both-finger stimuli are not included in this study. These responses were similar to the summed responses to separate right- and left-finger stimuli and showed a qualitatively identical perceived-unperceived difference.

Data analysis. We investigated the stimulus locking of the ongoing cortical activity by using the phase-locking factor (PLF). PLF quantifies the nonuniformity of a phase distribution. PLF ranges from 0 to 1 , with the value 1 indicating a $\delta$-function distribution and the values approaching 0 indicating a uniform distribution. For $N$ complex values $z_{i}, i=1 \ldots$ $N$, on the unit circle, the PLF is given by $N^{-1}\left|\Sigma z_{i}\right|$ (Sinkkonen et al., 1995). If values $z_{i}$ are uncorrelated and randomly drawn from a uniform distribution, the PLFs of different sets of $z_{i}$ obey the Rayleigh distribution $p(\lambda)=\lambda \sigma^{-2} \exp \left[-0.5 \lambda^{2} \sigma^{-2}\right]$, where $\lambda$ denotes the PLF for a given realization of $z_{i}, s=\lambda_{m}(0.5 \pi)^{-0.5}$, with $\lambda_{\mu}$ being the mean of the distribution. To assess the statistical significance of the PLFs obtained for our data, we used the Rayleigh test in which $\lambda_{\mu}$ is simply defined by the number of trials. The null hypothesis is that the samples were drawn from a uniform distribution. Because the Rayleigh distribution is a function of its mean $\left(\lambda_{m}\right)$ only, we found it appropriate to normalize the PLFs by $\lambda_{m}$ to express the strength of phase locking directly in a statistically meaningful manner. PLF $/ \lambda_{m}>1.95$ corresponds to $p<0.05$ and $\operatorname{PLF} / \lambda_{m}>$ 2.42 to $p<0.01$.

The presence of stimulus locking on a group level was evaluated using binomial statistics $\left(P_{\mathrm{B}}\right)$. The significance of the difference between the responses to perceived and unperceived stimuli was estimated with the Wilcoxon signed-rank test $\left(P_{\mathrm{W}}\right)$. To obtain a conservative control for the multiple statistical comparisons problem, we used Bonferroni's correction. For the data in Figure 2, $A$ and $C$, as well as in Figure 4, $A$ and $B$, the correction was based on the number of samples in the traces ( 270 and 90 and 126 and 126, respectively). For all topographical visualizations (see Figs. $2 B, 3 C, 5 B$ ), the correction was based on the number of visualized gradiometers (102). For all time-frequency representations (see Figs. $3 B, 4 B, 5 A, 6$ ), the correction for each frequency band was based on the number of the samples shown (240). The few cases in which we considered notable a result that only met the uncorrected significance criteria are explicitly commented on in Results.

The continuous phase of ongoing activity was obtained with two methods: for the results shown in Figures 2, 4, $A$ and $B$, and 7, the gradiometer signals were first filtered with a high-pass-low-pass finiteimpulse response-filter pair, defined by stopbands of 1 and $80 \mathrm{~Hz}$ and passbands of 2 and $40 \mathrm{~Hz}$. The phase $\phi(t)$ was then estimated with $\phi=$ $\arctan \left(s^{\prime} s^{-1}\right)$ by using the Hilbert transform to obtain the imaginary part $s^{\prime}(t)$ of the filtered data $s(t)$, where $t$ denotes time. For the timefrequency analyses in Figures 3-6, the data $x(t)$ were convolved with a family of Morlet wavelets $w\left(t, f_{0}\right), w\left(t, f_{0}\right)=A \exp \left(-t^{2} / 2 \sigma_{t}{ }^{2}\right) \exp \left(2 i \pi f_{0} t\right)$, where $\sigma_{t}=m / 2 \pi f_{0}, i$ is the imaginary unit, $m$ defines the compromise between time and frequency resolution, and $f_{0}$ is the center frequency of the wavelet. The convolution $X\left(t, f_{0}\right)=x(t) * w\left(t, f_{0}\right)$ gives a complex vector $X\left(t, f_{0}\right)$, of which the angle is the phase of the signal $x$ in a frequency band with a center frequency of $f_{0}$ and a frequency-domain SD of $\sigma_{f}=$ $f_{0} / m$. In this study, $m$ was 5.5, and the wavelet family was composed of center frequencies $f_{0, j}(j=1 \ldots 14)$, which were separated by $\sim 1 \sigma_{f}\left[f_{0, j}+\right.$ $\left.1-f_{0, j}=\left(\sigma_{f, j+1}+\sigma_{f, j}\right) / 2\right]$ and ranged from 5 to $55.5 \mathrm{~Hz}$. The time- and frequency-domain envelopes of the filters used in this study are shown in Figure $3 A$. For a $10 \mathrm{~Hz}$ wavelet, the time-domain $\mathrm{SD}\left(\sigma_{t}\right)$ is $87.5 \mathrm{~ms}$, and the time-domain envelope half-width at half-height is $103 \mathrm{~ms}$ (cf. Yamagishi et al., 2003). For the broadband filter, the half-width at half-height is $10.4 \mathrm{~ms}$. The time-frequency representations in Figures 3-6 were obtained with cubic interpolation into frequency steps of $0.5 \mathrm{~Hz}$.

The evoked responses from primary and secondary somatosensory cortices were obtained for each subject by averaging the responses within the pairs of MEG channels shown in Figure 2C (right) after taking the vector sum of the orthogonal gradiometers in each channel. The gradiometers over SI showed the maximum response amplitudes, and their location was essentially identical for all subjects (cf. Simões et al., 2003). The locations of gradiometers over SI and SII correspond to those identified earlier (Simões et al., 2003). The signal-to-noise ratio of the ERs to the very weak stimuli used in this study was insufficient to allow dipole modeling.

The nonstimulus-locked amplitude fluctuations were obtained by averaging across trials the amplitude envelopes given by Morlet wavelets and subtracting from each frequency band the mean baseline amplitude in a window of 200-300 ms before stimulus onset (Tallon-Baudry and Bertrand, 1999).

All analyses and visualizations were performed on a custom-made database analysis platform programmed in the LabView environment (National Instruments, Austin, TX).

\section{Results}

\section{Psychophysical performance}

We presented weak, constant-current electrical stimuli to the tips of subjects' index fingers with an intensity adjusted so that slightly $<50 \%$ of the stimuli were reportedly perceived during the minute before the onset of each data recording. The subjects underwent four $20 \mathrm{~min}$ MEG recordings during which the stimuli were presented semirandomly to left, right, or both index fingers with a random interstimulus interval ranging from 3 to $4 \mathrm{~s}$. The subjects were instructed to promptly report the detected stimuli with a twitch of the thumb of the stimulated hand. The thumb twitches were recorded with electromyography (see Materials and Methods) and were assumed to indicate that the stimuli were consciously perceived; however, we cannot exclude the 
A

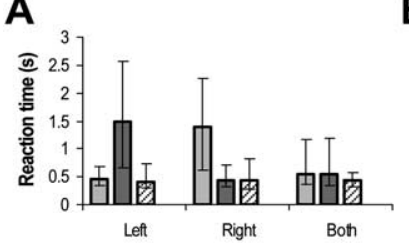

B
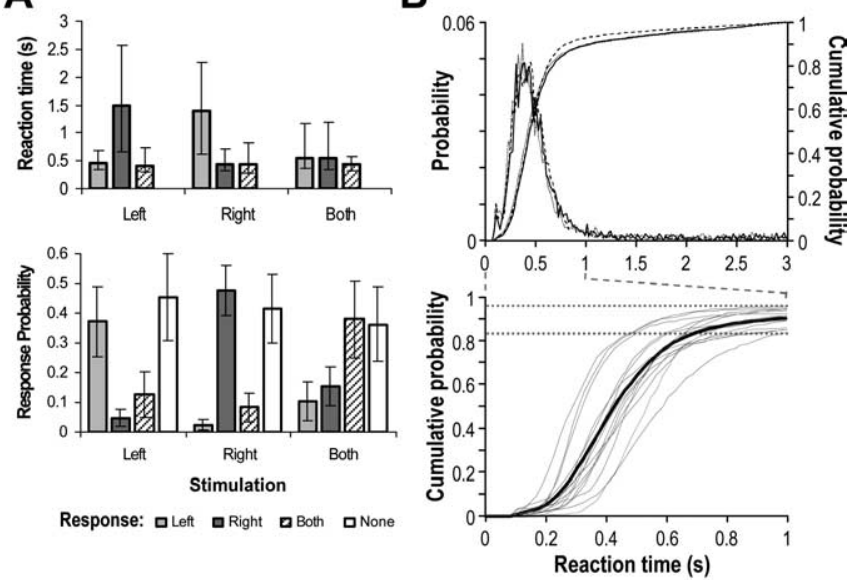

C

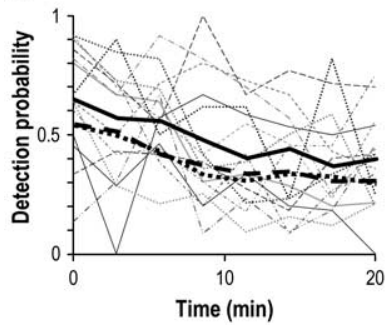

D

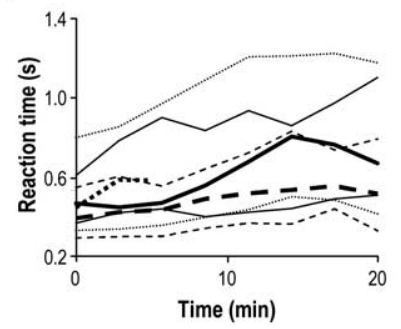

Figure 1. Characterization of subjects' psychophysical performance in the stimulusdetection task. $\boldsymbol{A}$, The histogram bars show the median reaction time (top) and the response probability (bottom) averaged across subjects for each stimulus- (on $x$-axis) response (indicated with bar texture) pair. The reaction time error bars indicate the average across subjects of the 16th and 84th percentiles of each subject's reaction-time distribution. The error bars of the detection probability indicate the SD (approximately corresponding to $\sim 16$ th and $\sim 84$ th percentiles) of the detection probability in the present subject population. $\boldsymbol{B}$, Top, The distribution of reaction times to left (dotted line), right (solid line), and both (dashed line) stimuli. Bottom, Thin gray lines indicate the cumulative reaction-time distribution of each subject averaged across the three stimulation conditions (note the expanded $x$-axis). The thick black line shows the average across subjects. C, The probability of detecting left (dotted line), right (solid line), and both (dashed line) stimuli was evaluated in $150 \mathrm{~s}$ segments and averaged across subjects and the four recording sessions. Thin gray lines show the data of each subject from the first recording session. $\boldsymbol{D}$, The median (thick lines) and 16th/84th percentiles (thin lines) of subjects' reaction times to left (dotted line), right (solid line), and both (dashed line) stimuli were averaged within the 150 s segments (as in C) and across the subjects and the four recording sessions.

possibility of preconscious or automatic reactions. Figure $1 \mathrm{~A}$ shows the mean detection probabilities ( \pm intersubject $\mathrm{SD}$ ) and the averaged median reaction times (RTs) with 16 and $84 \%$ confidence limits averaged across subjects. The subjects reacted fairly accurately; only 17,11 , and $26 \%$ of left, right, and both finger stimuli, respectively, were erroneously responded to (Fig. 1A). The RT distributions were clearly unimodal and showed median RTs of 460, 430, and $430 \mathrm{~ms}$ for left, right, and both finger stimuli, respectively (Fig. $1 B$, top). The intersubject variability in $\mathrm{RT}$, however, was considerable (Fig. $1 B$, bottom) and in line with the proposition that distinct processing routes to response initiation might be exploited by the fast- and slow-reacting subjects (cf. Jokeit and Makeig, 1994; Makeig et al., 1999). The presence of very fast responses $(\sim 200 \mathrm{~ms})$ in our data in some subjects raises a question about whether the initiation of motor reaction could occur also before or in parallel with the conscious sensory perception (cf. Jokeit and Makeig, 1994; Makeig et al., 1999), which suggests that also within individual subjects, distinct processing strategies might take place on different trials. Depending on the subject, 4-17\% of RTs exceeded 1 s (Fig. 1, horizontal dotted gray lines). According to signal-detection theory, the decision-making criteria may change when detecting weak signals because of the uncertainty of their presence (Wickens, 2002). The long-latency responses probably reflect the prolonged decision making or responding after doubt when the percept was ambivalent. Moreover, according to the theory, it is possible that some unperceived stimuli were actually weakly perceived but did not pass the criteria for decision making (Wickens, 2002). Although the presence of illusory percepts (false alarms) remains unclear because of the impossibility of separating the responses to real stimuli from the responses to imagined stimuli, their number is, in any case, very small. This is evident from the very small response probability between 1.5 and $3 \mathrm{~s}$ after stimulus onset (Fig. $1 \mathrm{~B}$, top).

We did not find significant differences in psychophysical performance among the four $20 \mathrm{~min}$ recordings (data not shown; $p>0.05$ ), but during the course of every 20 min recording, presumably because of fatigue, the detection probabilities decreased moderately ( $p<0.001$; first half vs second half) (Fig. $1 C$ ) and the reaction times became slightly longer $(p<0.05)$ (Fig. $1 D)$. Nevertheless, the dynamic variability of these behavioral measures in individual subjects was, throughout each recording, larger than the effects of fatigue (Fig. 1C,D), indicating that the psychophysical performance was also influenced by factors on smaller time scales (such as fluctuations of arousal) (LinkenkaerHansen et al., 2004). Finally, we estimated whether the success in stimulus detection influenced the detection probability of subsequent stimuli in the present data. The data showed that, when compared with randomized surrogate data in short $40 \mathrm{~s}$ windows, detection was more probable $[2.7 \pm 0.8 \%$ (mean \pm SEM) $]$ when the previous stimulus was detected and, conversely, less probable $(4.2 \pm 1.2 \%)$ when the previous stimulus was missed. This suggests that fluctuations in attention or in short-term memories of preceding stimuli contributed to the brain states facilitating perception.

\section{Broadband stimulus locking}

To characterize the neural activity related to the consciously perceived and unperceived somatosensory stimuli with high temporal resolution, we quantified the phase locking of the ongoing broadband $(1-80 \mathrm{~Hz})$ neural activity to these stimuli. This kind of a stimulus-locking analysis reveals whether the recorded signals at a given latency show a nonrandom phase relationship to the presented stimuli across the trials (Sinkkonen et al., 1995). Because the phase-locking factor is amplitude independent, it is robust to large-amplitude artifacts and may reveal smallamplitude but well locked events better than the conventional evoked responses that mix amplitude and phase information. We found that, after the stimulus presentation, neural activity phase locked more strongly and persistently to the consciously perceived stimuli than to the unperceived stimuli (Fig. 2 A). Notably, the difference between the perceived and unperceived stimuli reached statistical significance as early as $30 \mathrm{~ms}$ after stimulus onset (Fig. 2 A). For both consciously perceived and unperceived stimuli, the stimulus-locked activities were first observed over the contralateral SM region; for the perceived stimuli only, the stimulus locking thereafter spread rapidly to the frontal, parietal, and ipsilateral SM regions (Fig. 2B) (see supplemental video 1, available at www.jneurosci.org as supplemental material). Stimulus locking to the perceived stimuli was significant at $40 \mathrm{~ms}$ over frontal cortical regions and at $\sim 70 \mathrm{~ms}$ over the parietal and ipsilateral SM regions (Fig. 2C). Interestingly, there was also significant stimulus locking of neural activity before the onset of the 


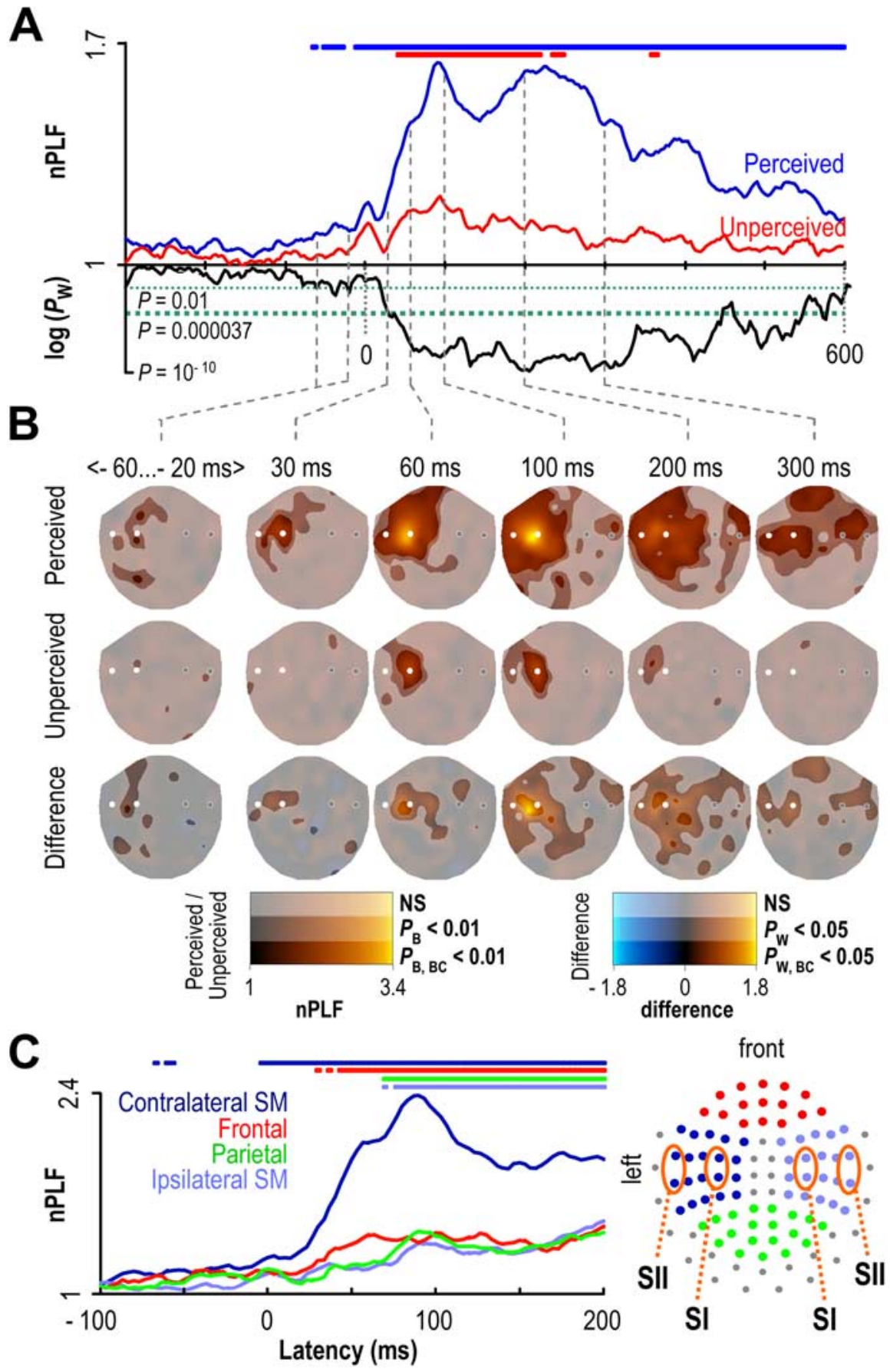

Figure 2. Somatosensory conscious perception is reflected in the early stimulus-locked responses. $\boldsymbol{A}$, The normalized PLF (nPLF), quantifying the phase locking of broadband $(1-80 \mathrm{~Hz}$ ) activity to perceived (blue) and unperceived (red) stimuli, and the statistical significance (Wilcoxon signed-rank test; $P_{\mathrm{w}}$ ) of their difference (black), averaged across all subjects and MEG gradiometers. The green dotted lines denote the $P_{\mathrm{W}}<0.01$ significance level (small dots, uncorrected; large dots, Bonferroni's corrected). The bars above the waveforms indicate the time periods of significant phase locking (binomial statistics; $P_{\mathrm{B}, \mathrm{BC}}<0.01$, where $\mathrm{BC}$ indicates Bonferroni's correction). $B$, Topographies of phase locking of ongoing activities to right-hand stimuli. The MEG helmet covering the entire scalp is flattened, and the anterior direction is upward. The nPLFs, averaged across subjects, are shown on the color scale. The transparency levels indicate the significance of the phase locking [not significant (NS), $P_{\mathrm{B}}$, and $P_{\mathrm{B}, \mathrm{BC}}$ ] and that of the perceived- unperceived difference ( $N S, P_{\mathrm{W}}$, and $P_{\mathrm{W}, \mathrm{BC}}$ ). The white and gray dots show the approximate locations of contralateral and ipsilateral, respectively, SI (medial) and SII (lateral). C, The phase locking to the detected stimuli averaged across gradiometer selections over the somatosensory (contralateral, blue; ipsilateral, light blue), frontal (red), and parietal (green) regions. The bars denote $P_{\mathrm{B}, \mathrm{BC}}<0.01$, as in $\boldsymbol{A}$. Each individual gradiometer pair in these selections is shown with a correspondingly colored dot in the topography (right). The orange lines surround the selections of two gradiometer pairs that were used to inspect signals from SI and SII in Figures 5 and 6. subsequently perceived stimuli but not before the onset of the unperceived stimuli (Fig. $2 A, B$ ), showing that the phase of the cortical state may bias stimulus detection. Interestingly, the spatial topology of this prestimulus activity was fairly similar to the hemodynamic pattern of activation observed during somatosensory anticipation (Carlsson et al., 2000).

\section{Time-frequency analysis of the phase locking of ongoing activity to sensory stimuli}

The analysis of stimulus locking of broadband phase (Fig. 2) allows the evaluation of the precise time course of the phaselocked events but does not disclose the spectral characteristics of the underlying activities (Fig. 3A, black lines). The eventrelated responses are known to comprise both evoked components but also stimulus locking of neuronal oscillations in distinct frequency bands (Tallon-Baudry et al., 1999; Makeig et al., 2002; Simões et al., 2003). Therefore, we also examined stimulus locking in the time-frequency domain using Morlet wavelets. Such analysis sheds light on the frequency content of the response but with greatly compromised temporal resolution (Fig. 3A, colored lines) (see Materials and Methods) that makes, for instance, the determination of oscillation onsets ambiguous. We found that, in all frequency bands and in all regions, the phase locking to the consciously perceived stimuli was stronger and spatially more widespread than the phase locking to the unperceived stimuli (Fig. $3 B$ ) (see supplemental video 2 , available at www.jneurosci.org as supplemental material). The responses to the perceived stimuli were dominated by widespread stimulus locking of the $\theta-(4-8 \mathrm{~Hz})$ and $\alpha$ - (8-14 Hz) frequency-band phases (Fig. $3 B$ ) not only in somatosensory but also in frontal and parietal areas. In addition, in the contralateral SM region, we also observed the phase locking of the $\beta$ - (14-28 $\mathrm{Hz})$ and low- $\gamma-(28-40 \mathrm{~Hz}$; hereafter referred to as $\gamma$-) frequency bands, but in the frontal, parietal, and ipsilateral SM regions, the phase locking of these bands to the perceived stimuli was weak or absent, respectively (Fig. 3B,C). The phase locking to the unperceived stimuli, in contrast, involved a $\theta$-band component and, in the contralateral SM region, a $\beta$-band component as well (Fig. 3B).

These data thus show that the largescale $\alpha$-band stimulus locking, prominent for the consciously perceived stimuli and mostly absent for the unperceived stimuli, is the most salient qualitative feature dis- 
A
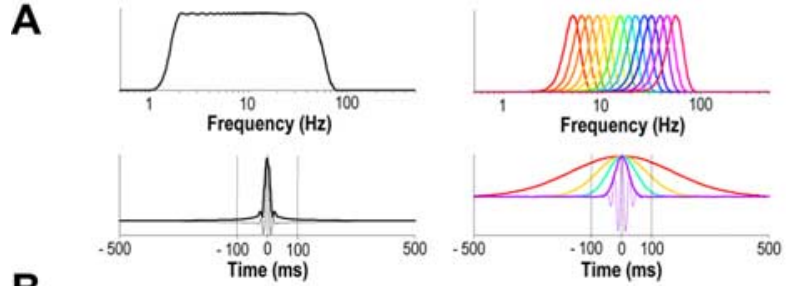

B
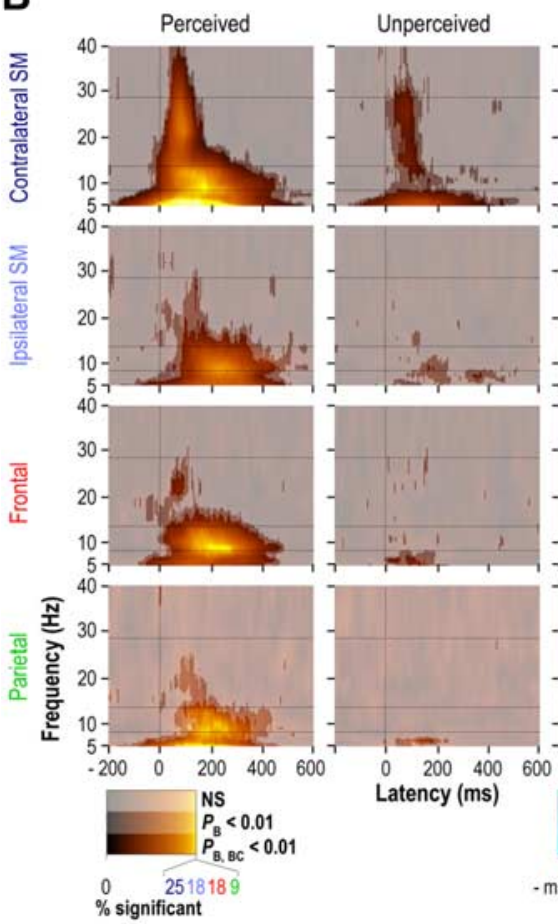

Difference
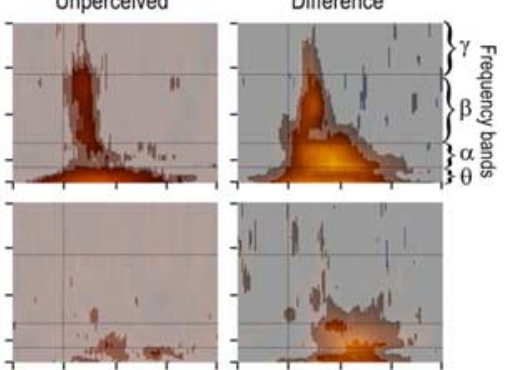

C
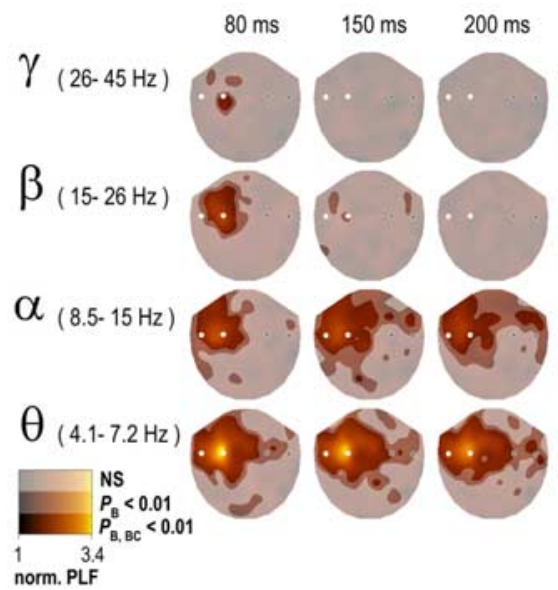

$300 \mathrm{~ms}$

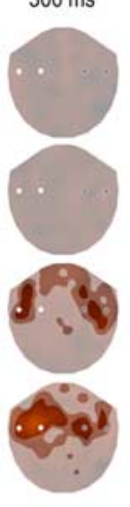

Figure 3. The stimulus locking of large-scale $\alpha$-frequency-band oscillations is strongly correlated with conscious perception. $\boldsymbol{A}$, Schematic frequency-domain (top panels) and timedomain (bottom panels) representations of the filters used in this study. Left, Black lines, Broadband filter composed of high- and low-pass finite-impulse response filters. Right, Colored lines, A bank of 14 Morlet wavelets. The center frequencies of the examples chosen for the bottom panel are $5 \mathrm{~Hz}$ (red), $\sim 10 \mathrm{~Hz}$ (yellow), $\sim 20 \mathrm{~Hz}$ (cyan), and $\sim 40 \mathrm{~Hz}$ (purple). Note the considerable width of the less than $\sim 10 \mathrm{~Hz}$ wavelets when interpreting the time-frequency representations below. $\boldsymbol{B}$, The color scale of the time-frequency representations shows the proportion of gradiometers with statistically significant phase locking (averaged across subjects). The transparency levels indicate the significance of phase locking [not significant (NS), $P_{\mathrm{B}^{\prime}}$ and $P_{\mathrm{B}, \mathrm{BC}}$ ] and that of the perceived- unperceived difference (NS, $P_{\mathrm{W}}$ and $\left.P_{\mathrm{W}, \mathrm{BC}}\right)$. $C$, Topographies of phase locking to perceived right-hand stimuli in different frequency bands $(\theta, \alpha, \beta$, and $\gamma)$. For each band, averages of the topographies of two wavelet frequencies are shown. The frequency ranges given next to the band symbols indicate -1 and +1 SD from the lower and higher, respectively, wavelet center frequencies. norm. PLF, Normalized PLF. tinguishing conscious from unconscious processing. This was best exemplified in the contralateral SM, in which the responses to unperceived stimuli lacked significant $\alpha$-band phase locking, although they were strong enough to reveal both $\theta$ - and $\beta$-band phase locking. In addition to the clear perception specificity of $\alpha$-band oscillations, the $\gamma$-band stimulus locking in the contralateral SM also appeared to be much stronger for the perceived than for the unperceived stimuli, although the perceived-unperceived difference did not reach the Bonferroni-corrected significance level. It should also be noted that strong conclusions, with regard to specificity of the $\gamma$-band component to conscious perception, cannot be drawn because of its poor signal-to-noise ratio.

\section{Time-frequency analysis of amplitude dynamics}

The processing of consciously perceived and unperceived somatosensory stimuli thus diverges already during early stimuluslocked processing. We then addressed the correlates of stimulus perception in the amplitude of nonstimulus-locked activities (for review, see Tallon-Baudry and Bertrand, 1999). All stimuli were followed by clear and prolonged suppression of the amplitudes of the $\alpha$-, $\beta$ - (Nikouline et al., 2000; Penna et al., 2004), and $\gamma$-frequency bands. In all regions, these amplitude decreases were larger for the perceived than for the unperceived stimuli (Fig. $4 A)$. Interestingly, the $\alpha$-band amplitude decrease began in the same latency range (200-300 ms after stimulus onset) in which the $\alpha$-band stimulus locking began to attenuate (Fig. 3B). Correspondingly, the onset of $\beta$ - and $\gamma$-frequency-band amplitude decreases over the contralateral SM also matched well with the offset of stimulus locking in these frequency bands. However, we did not find significant increases in the $\gamma$-frequency-band amplitude (i.e., induced $\gamma$ oscillations) anywhere in the interval from stimulus onset to the behavioral response (cf. Tallon-Baudry and Bertrand, 1999). However, over SM and frontal regions, we observed a prominent increase in $\theta$-frequency-band amplitude that was greater for the consciously perceived than for the unperceived stimuli (Fig. 4A,B). The time course and spatial topography of this $\theta$ amplitude increase, however, were very similar to those of the stimulus-locked $\theta$ component, which suggests that the enhanced amplitude and stimulus locking are reflections of the same evoked process.

Together, in our data, the rapid cascade of stimulus-locked processing was followed by a suppression of the amplitude of ongoing activity in all frequency bands, except for the $\theta$ band. In the framework in which increases in the amplitude of nonstimulus-locked $\beta-/ \gamma$-band oscillations are interpreted as a signature of transient cognitive processes (Tallon-Baudry and Bertrand, 1999), the amplitude drop in $\alpha$-, $\beta$-, and $\gamma$-frequency bands suggests that later, nonstimulus-locked processing might be less essential in the present task.

\section{Stimulus locking and amplitude dynamics over primary and secondary somatosensory cortices}

To compare the present findings with previous studies on somatosensory processing, we also computed the conventional, broadband averaged ERs of SI and SII (see Materials and Methods) (Simões et al., 2003) contralateral to the stimulated finger. Over SI, the ER comprised distinct components at $\sim 30,60$, and $100 \mathrm{~ms}$ from stimulus onset (Fig. 5A) (Hari et al., 1993; Mauguiere et al., 1997). Over SII, the first major component was found at $100 \mathrm{~ms}$ from stimulus onset (Hari et al., 1993; Mauguiere et al., 1997; Simões et al., 2003), but it was preceded by small peaks at $\sim 30$ and $60 \mathrm{~ms}$ (Karhu and Tesche, 1999). Each of these components was larger for the perceived than for the unperceived stim- 
A

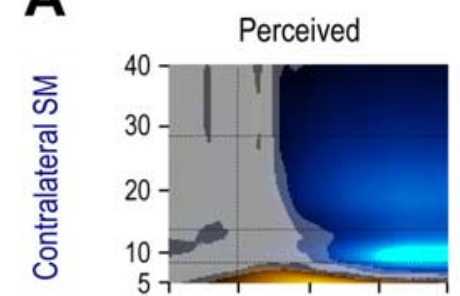

$5 \uparrow \longrightarrow$

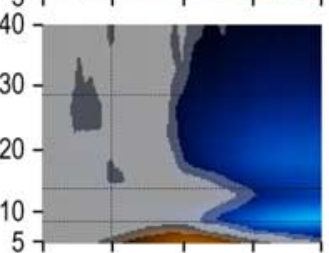

$57+\frac{2}{10-1}$
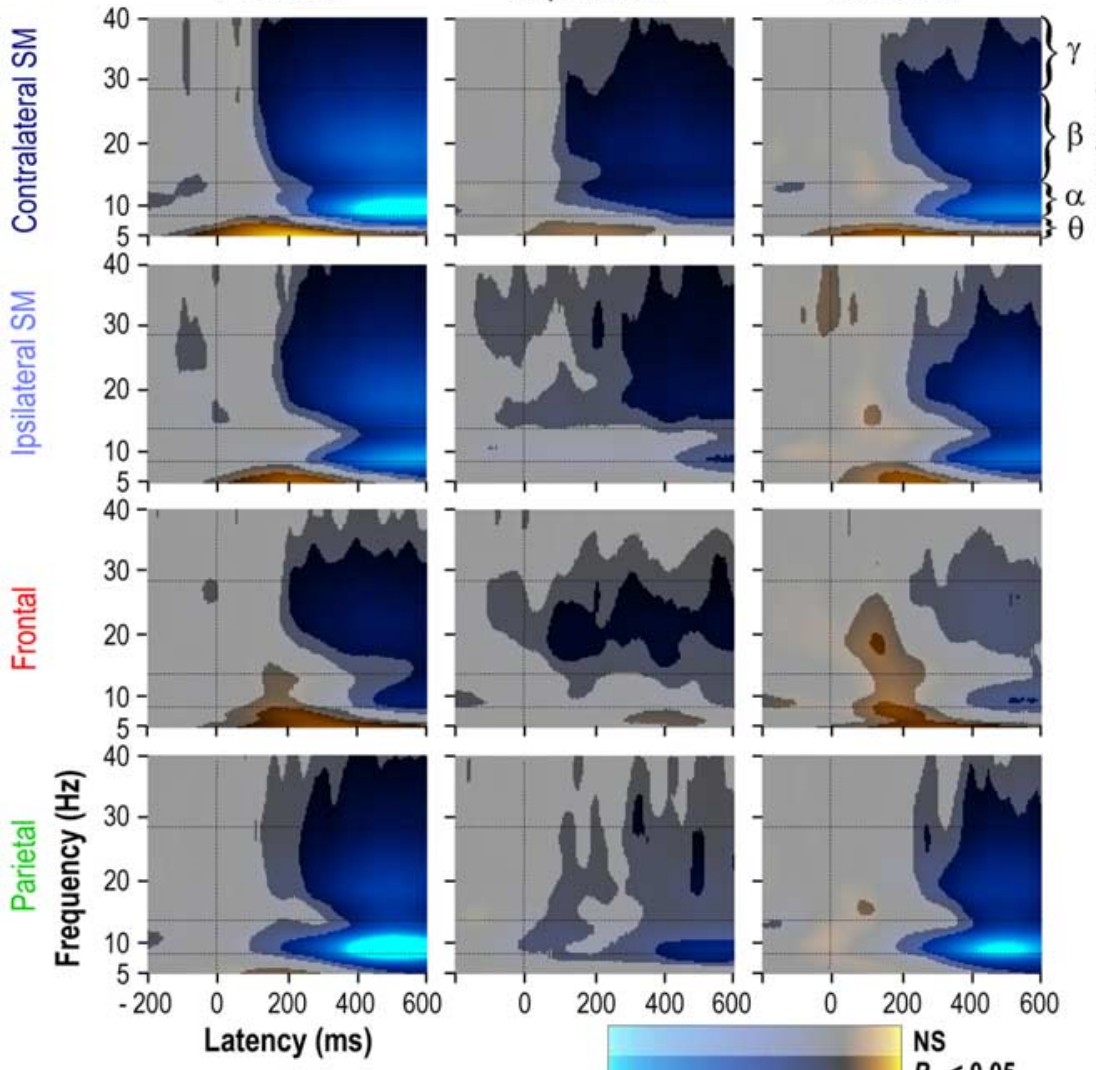

$P_{w}<0.05$

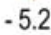

$P_{\mathrm{W}, \mathrm{BC}}<0.05$

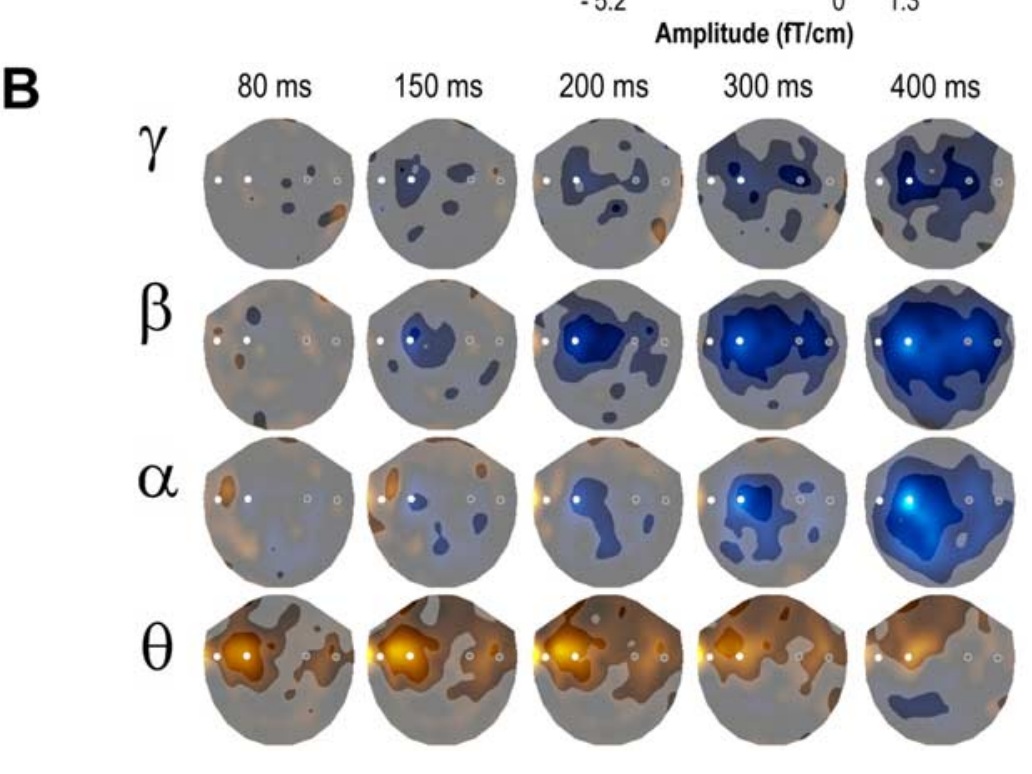

$0 \quad 1.3$

Amplitude ( $\mathrm{fT} / \mathrm{cm})$
Figure 4. The amplitudes of ongoing $\alpha, \beta$, and $\gamma$ oscillations are suppressed by both perceived and unperceived stimuli widely over the cortex. $A$, The color scale of the time-frequency representations shows the oscillation amplitude after subtraction of the baseline $(-300$ to $-200 \mathrm{~ms})$. The transparency levels indicate the significance of amplitude-baseline difference for perceived and unperceived stimuli as well as that of the perceived- unperceived difference [not significant (NS), $P_{\mathrm{W},}$ and $P_{\mathrm{W}, \mathrm{B}}$ ]. $\boldsymbol{B}$, Topographies of the poststimulus amplitude changes in different frequency bands (as in Fig. 3C). The color scale is the same as that in $\boldsymbol{A}$.

(Fig. 5A) (note that, for the perceived-unperceived difference; $0.00008<P_{\mathrm{W}}<$ $0.01)$. The timing of these peaks, as well as that of the poststimulus $\sim 30,60$, and 100 $\mathrm{ms}$ peaks, is suggestive of low $\gamma$-frequency-band $(\sim 30 \mathrm{~Hz})$ rhythmicity, but because these plots show the amplitude envelope of the broadband activity, the presence of $\gamma$-band oscillations per se cannot be inferred directly.

For SI and SII, we also evaluated the broadband phase locking of neural activity to the stimuli. As expected, over SI, this stimulus locking showed a clear difference between the perceived and unperceived stimuli from $30 \mathrm{~ms}$ onward (Fig. 5B). The stimulus locking over SII was very weak for the unperceived stimuli. These data suggest that, although the processing of unperceived stimuli clearly involved SI, the activation of SII was, at most, very weak. Note that the ER (Fig. 5A) and stimulus-locking (Fig. 5B) analyses here are directly comparable because of the identical broadband filtering.

We also examined the time-frequency characteristics of stimulus locking over contralateral and ipsilateral SI and SII. The phase locking to the perceived stimuli over contralateral SI involved all examined frequency bands; however, over SII, no $\gamma$-band stimulus locking was observed (Fig. 5C). Furthermore, over ipsilateral SI and SII, only $\theta$ - and $\alpha$-band stimulus locking was significant. The responses to unperceived stimuli over contralateral SI were composed only of $\theta$ - and $\beta$-band components, and, furthermore, significant phase locking was not observed over SII (Fig. 5C) or over ipsilateral SI or SII.

Over the primary and secondary somatosensory cortices, the pattern of poststimulus amplitude dynamics (Fig. 6) was similar to that observed for the somatomotor region (Fig. 4). In addition to the rapid amplitude suppression in $\alpha-, \beta$-, and $\gamma$-frequency bands below the mean baseline level, a brief increase in $\gamma$-band amplitude was observable, albeit below the Bonferroni-corrected significance level, over the primary somatosensory cortex for the consciously perceived stimuli at $\sim 50-100 \mathrm{~ms}$ from stimulus onset, coinciding with stimulus locking in the $\gamma$ band (Fig. 6) (contralateral SI; perceived). Notably, also just before stimulus onset, the $\beta$ - $/ \gamma$-band amplitude was greater for the perceived than for the unperceived uli; however, the early components over SII did not exceed the Bonferroni-corrected significance level. Intriguingly, small peaks of prestimulus activity were observable at $\sim 30$ and $60 \mathrm{~ms}$ before the onset of the perceived stimuli over SI and at $\sim 30$ ms over SII stimuli (Fig. 6) (contralateral SI; unperceived and difference; note again, only $\left.P_{\mathrm{W}}<0.05\right)$. This finding is in line with data showing that prestimulus $\gamma$-band oscillations facilitate subsequent processing (Fries et al., 2001a). 
Correlation of early stimulus locking with reaction time

To find out whether the early ( $<100 \mathrm{~ms})$ stimulus-locked and perception-related processing has an impact on the subsequent reaction time, we divided the perceived trials evenly into three categories: fast (the fastest third of RTs) (Fig. 7, thick red lines), mid (the middle third of RTs) (Fig. 7, thick green lines), and slow (the slowest third of RTs) (Fig. 7, thick blue lines). The phase locking of ongoing activity to the stimuli was quantified after broadband filtering as above in Figures 2 and 5 . To rule out the possibility that slow fluctuations in attention or cortical excitability could have contributed jointly to differential stimulus locking and reaction time, we also collected three categories of responses to the unperceived stimuli. The number of trials in these categories was equal to that in fast, mid, and slow, and, moreover, the trials were selected so that they were as near as possible to the corresponding perceived trials (Fig. 7, thin lines). First, these data show that the fast reaction times are clearly associated with strong phase locking of the ongoing activity to the stimuli. Second, in contrast to the fastest two thirds (Fig. 7, thick red and green lines) of responses that were preceded by rapid and widespread stimulus locking over somatosensory, frontal, and parietal regions, the slowest third (Fig. 7, thick blue lines) of responses involved essentially negligible early stimulus locking over SII, frontal, and parietal regions. Because the responses to unperceived stimuli were essentially identical across fast $/ \mathrm{mid} /$ slow categories, slow fluctuations are unlikely to account for the intercategory differences observed here in the responses to the perceived stimuli. Hence, in light of these data, early large-scale stimulus locking of ongoing activity appears to be essential for fast behavioral reaction.
Figure 5. The evoked responses and phase locking to the consciously perceived and unperceived stimuli over SI and SII (Fig. 2C). $A$, The amplitude envelopes of conventional evoked responses over contralateral SI and SII to perceived (blue) and unperceived (red) stimuli and the statistical significance $\left(P_{\mathrm{w}}\right.$ and $P_{\mathrm{W}, \mathrm{BC}}$ ) of their difference (black). nPLF, Normalized PLF. $B$, Broadband stimulus locking over contralateral SI and SII to the perceived (blue) and unperceived (red) stimuli, and the statistical significance $\left(P_{\mathrm{w}}\right.$ and $\left.P_{\mathrm{W}, \mathrm{BC}}\right)$ of their difference (black), as in Figure 2A. C, Time-frequency representations of the strength of stimulus locking over contralateral and ipsilateral SI and SII averaged across subjects. The significance levels are indicated as in Figure 3B. NS, Not significant.
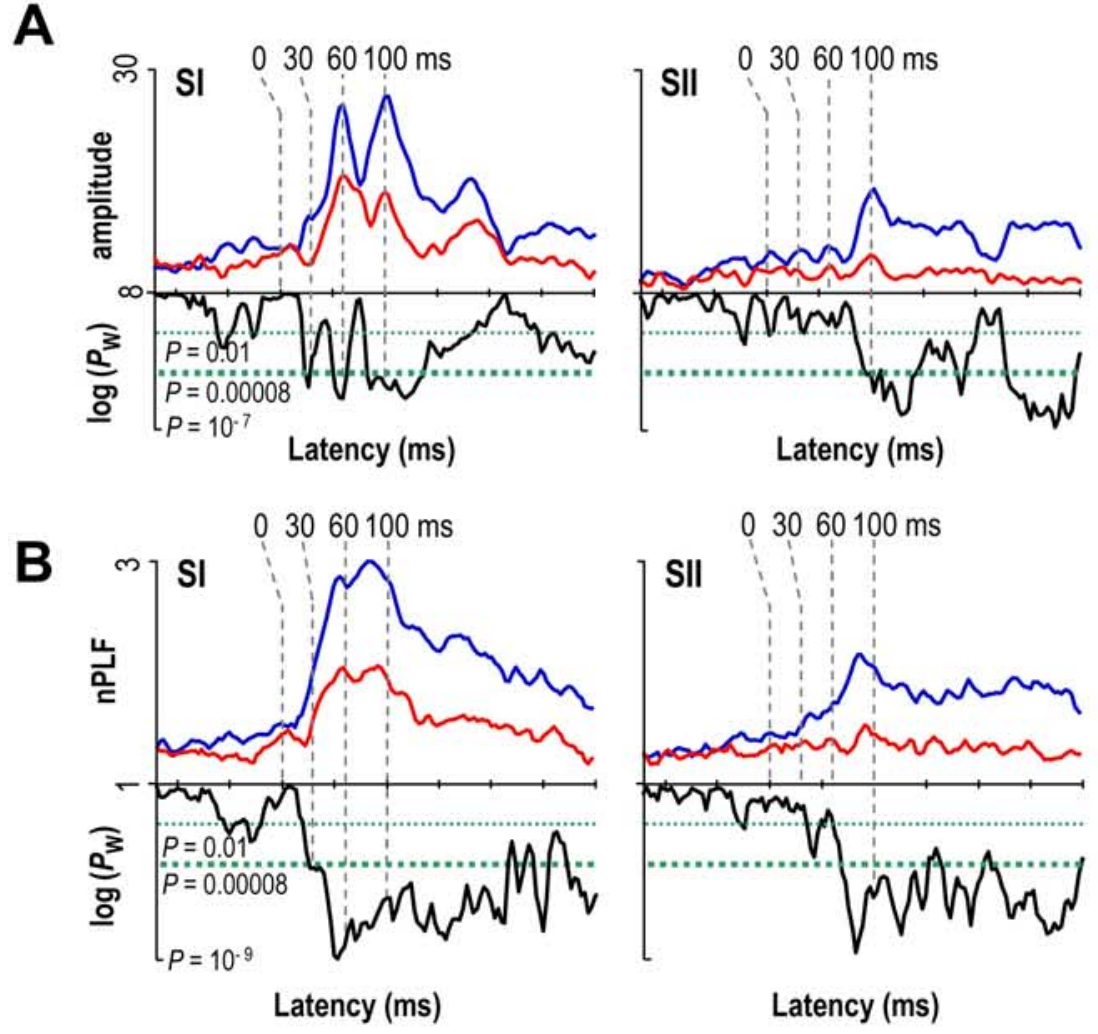

C
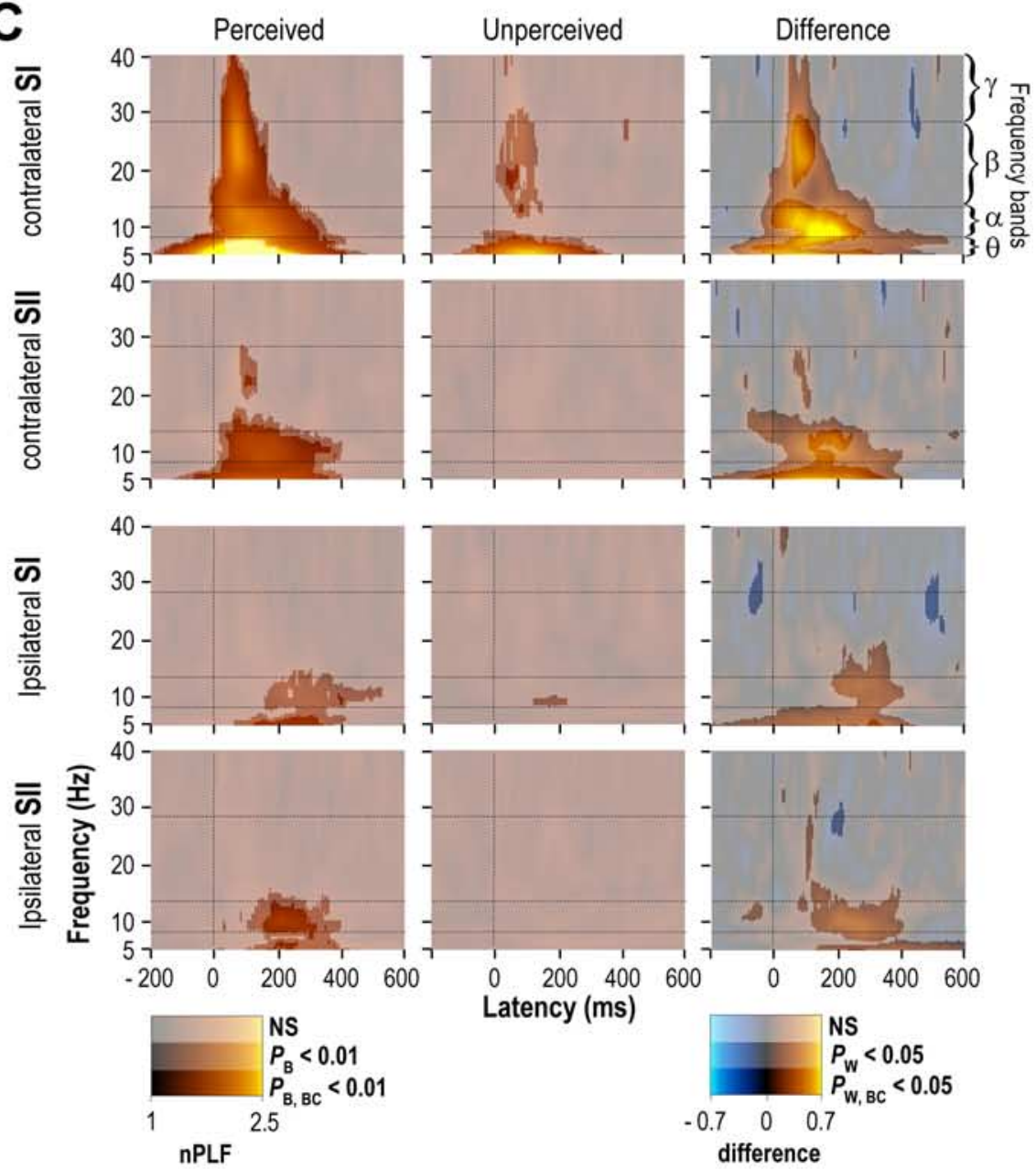

difference 


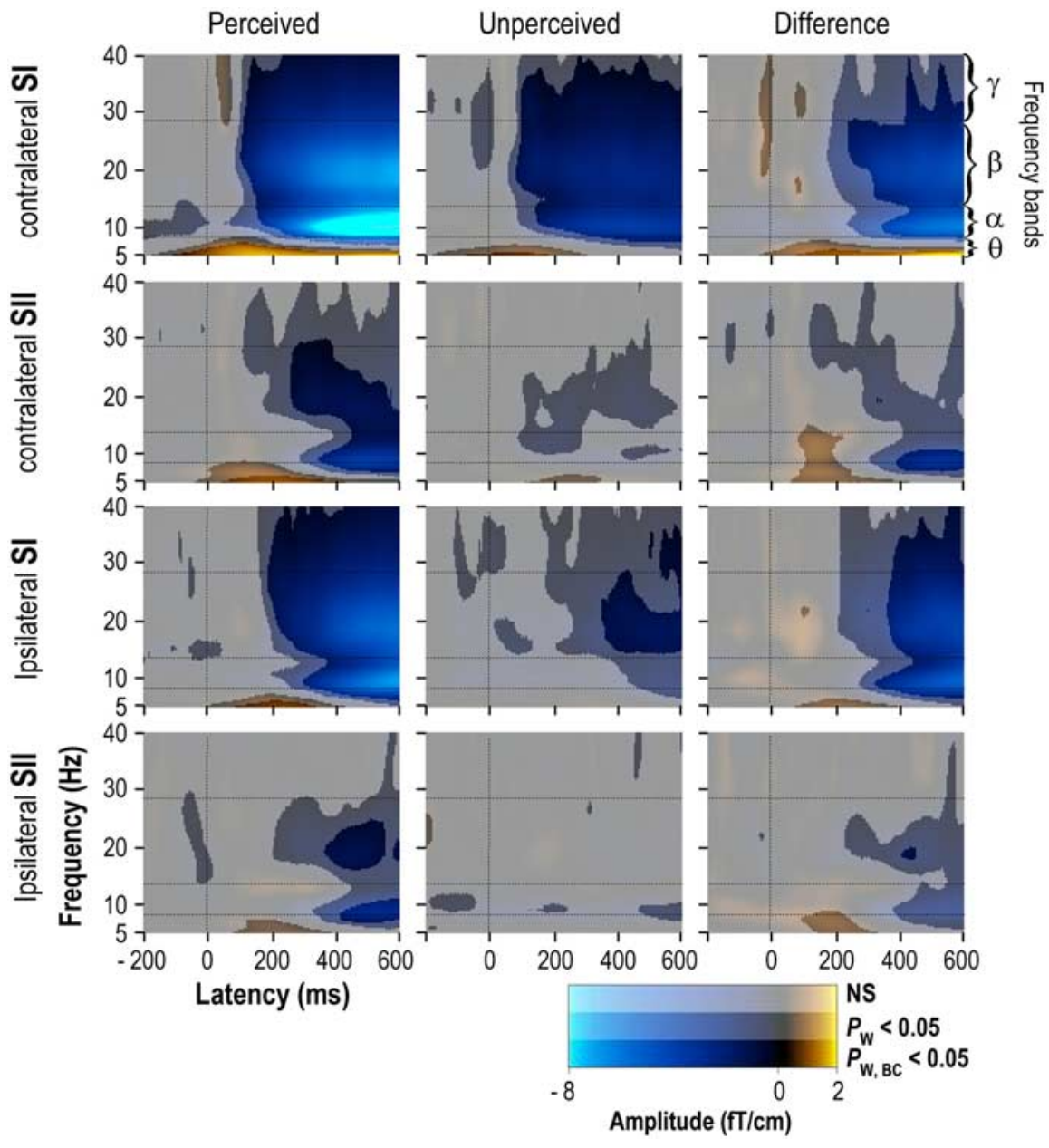

Figure 6. Amplitude dynamics of ongoing oscillations over SI and SII. The baseline and the significance levels are as in Figure 4 . NS, Not significant.

\section{Discussion}

We showed here that neural activity in somatosensory cortex, as well as in areas higher in the processing hierarchy, phase locked robustly to consciously perceived stimuli and that the phase locking to unperceived stimuli was weak and restricted to the SM region. The neural processing of these stimuli already diverged at $30 \mathrm{~ms}$ after stimulus onset. Observations of this divergence before $100 \mathrm{~ms}$ after stimulus onset add on to previous studies that have demonstrated detection sensitivity of the N1 component both in human (Parasuraman and Beatty, 1980; Pins and Ffytche, 2003) and in rhesus monkey (Kulics, 1982) ERs and also in later $(>100$ $\mathrm{ms}$ ) activities in human (Meador et al., 2002) and primate (Supèr and Lamme, 2001) primary sensory cortices. Interestingly, frontal and parietal regions, known to have a critical role in attention (Coull, 1998; Kastner and Ungerleider, 2000) and consciousness (Dehaene et al., 2001; Rees et al., 2002; Marois et al., 2004), were coactive with the sensory regions from 80 to $150 \mathrm{~ms}$ from stimulus onset. The formation of a sensorifrontoparietal network has been suggested to be essential for conscious processes (Rees et al., 2002; Zeman, 2004), but its activation at such an early latency is surprising (Dehaene et al., 2001; Pins and Ffytche, 2003). The correlation of this early stimulus locking with fast behavioral responses (Fig. 7) suggests that the activity of the underlying widespread and coherent neuronal assembly is likely to have a significant impact on consecutive neural processing.

Together, short reaction times, early activation of the fronto- parietal network, and the subsequent multiband amplitude suppression suggest that neural processing underlying conscious perception might already begin during an interval from 30 to $100 \mathrm{~ms}$ after stimulus onset.

\section{Contribution of prestimulus and early poststimulus neural activity to stimulus detection}

The phase locking to perceived and unperceived stimuli diverged as early as $30 \mathrm{~ms}$ after stimulus onset, although the stimulus current was identical for these stimuli, suggesting that neural activity in the prestimulus period contributes to the "perceptual fate" of the stimuli. We showed previously that, indeed, the prestimulus amplitude of ongoing oscillations has a strong influence on behavioral performance (Linkenkaer-Hansen et al., 2004). The present data (Figs. $2 A, B, 4 A, B$ ) also showed that the phase of brief cortical states before stimulus onset may bias subsequent perception. This is in line with studies suggesting that synchronous neural assemblies in the prestimulus period facilitate subsequent processing and reflect the top-down activation or priming of stimulus representations (Engel and Singer, 2001; Fries et al., 2001a; Supèr et al., 2003).

In contrast, attention may also modulate poststimulus neural activity already in the primary somatosensory areas (GarciaLarrea et al., 1991; Steinmetz et al., 2000) and enhance evoked responses as early as 20-50 ms after stimulus onset (Garcia-Larrea et al., 1991; Tiitinen et al., 1993; Fries et al., 2001b). Hence, it is likely that both the prestimulus and poststimulus top-down attentional effects contribute to the early divergence of conscious from unconscious processing in our data. Together, the probability of perception is likely to be influenced by intertwined phenomena at many temporal scales: by fatigue and changes in arousal, by fluctuations in attention, by variable accuracy of selective attention and of shortterm memories of preceding stimuli, by intermittent prestimulus cortical states, and finally, by various factors in poststimulus neural processing ranging from early top-down modulation to uncertainty in decision making.

\section{$\boldsymbol{\alpha}$-Frequency-band oscillations}

The large-scale stimulus locking of the $\alpha$-frequency-band oscillations, extending to the frontal and parietal regions, was found almost exclusively for the perceived stimuli. At first glance, this is at odds with the traditional view of large-amplitude $\alpha$ oscillations reflecting cortical idling. This "idling hypothesis" has emerged from studies that showed a transient decrease in $\alpha$-band amplitude in response to sensory stimuli or movements (Pfurtscheller and Lopes da Silva, 1999). Later work has shown that parietooccipital $\alpha$ oscillations in electroencephalograms (EEGs) are larger in amplitude over the hemisphere ipsilateral to the attended visual hemifield (Worden et al., 2000) and that they are also strengthened by attention directed to the auditory modality 

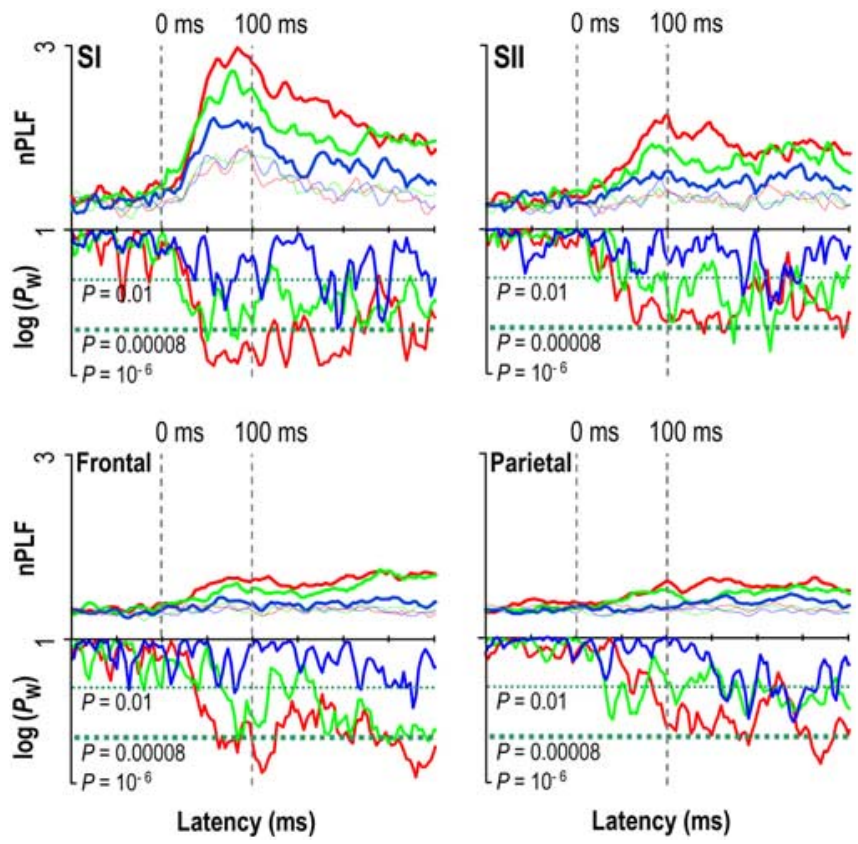

Figure 7. The strength of broadband stimulus locking is correlated with reaction time. The responses were pooled into three categories for each subject according to the reaction time and then averaged across subjects as well as within contralateral SI, SII, frontal, and parietal channel selections (Fig. 2C). The mean phase locking of ongoing activities to the perceived stimuli [normalized PLF (nPLF)] is shown with thick lines colored according to the reaction time category (fast, red; mid, green; slow, blue). The thin lines show the responses to equal numbers of unperceived stimuli picked nearby the corresponding perceived stimuli. The $\log \left(P_{\mathrm{W}}\right)$ scale shows the statistical significance of the perceived-unperceived difference (as in Fig. 2A).

(Fu et al., 2001). Hence, $\alpha$ oscillations have been interpreted to signal the suppression of unattended stimuli. In line with these previous data, the $\alpha$-band amplitude in our data was strongly "suppressed" after the detected stimuli. In the idling framework, this $\alpha$ "desynchronization" would indicate a period and region of active cortical stimulus processing. However, as the amplitude decrease followed massive stimulus locking, one must question whether an amplitude decrease is a signature of a process that is unrelated to task execution per se (and hence suppressed). The amplitude decrease could reflect a continuation of the physiological processes underlying the stimulus-evoked components and the "phase reset" of ongoing oscillations (Makeig et al., 2002, 2004). Nevertheless, because little is known about the physiological substrates of these macrolevel phenomena, the extent to which stimulus locking and amplitude changes reflect distinct processes remains unclear.

Indeed, a growing body of evidence suggests that $\alpha$-band oscillations may, in fact, be involved in higher cognitive functions such as attention and working memory. A recent MEG study showed that the amplitude of early $(\sim 0-200 \mathrm{~ms}$ after stimulus onset) $\alpha$ oscillations in the visual cortex is larger for the attended than for the unattended stimuli (Yamagishi et al., 2003). In addition, stimulus- and nonstimulus-locked $\alpha$-band oscillations are stronger during short-term memory retention than during baseline in intracranial (Halgren et al., 2002) and MEG (Jensen et al., 2002) recordings. Interestingly, nonstimulus-locked $\alpha$-frequency-band neural synchrony in the cat cortex was prominent in the responses to expected objects but not in those to novel objects, clearly indicating a role for $\alpha$-band synchrony in top-down modulation (von Stein et al., 2000). Finally, temporal intervals corresponding to $\alpha$-frequency-band periodicity have been observed in a number of psychophysical studies on cognitive integration windows (for review, see Van Rullen and Koch, 2003). Attention has been suggested to be a prerequisite for both consciousness and working memory (Lamme, 2003) and, evidently, $\alpha$-frequency-band oscillations are involved in all three of these. Additional experimental data are required to unbundle the neuronal mechanisms and specific functional roles of the $\alpha$-band rhythmicity in these processes.

\section{$\boldsymbol{\beta}$ - and $\boldsymbol{\gamma}$-frequency-band oscillations}

$\beta$-Band stimulus locking was stronger for the perceived stimuli than for the unperceived stimuli, although it was also clearly present in the responses to the unperceived stimuli (Figs. 3B, 4C). In the $\gamma$ band, significant stimulus locking was observed for the perceived but not for the unperceived stimuli. These findings are in line with previous studies showing a correlation between conscious perception and $\gamma$-band activity. In strabismic cats, perceived gratings induce greater nonstimulus-locked $\gamma$-band spike synchrony than those that were not perceived (Fries et al., 1997). Also in humans, the strengthening of $\gamma$-band synchrony between anatomically distinct regions both in intracortical (Meador et al., 2002) and in EEG (Rodriguez et al., 1999) data is correlated with stimulus perception. In this context, the perception sensitivity of "sensory-areas-restricted" $\gamma$-band and that of the more widespread $\beta$-band stimulus locking in our data is compatible with suggestions that these oscillations have a role in intra-areal and interareal feature binding, respectively (Castelo-Branco et al., 1998; Singer, 1999). However, in our data, we did not observe nonstimulus-locked amplitude increases (i.e., induced oscillations in the $\beta$ - or $\gamma$-frequency bands), which have been suggested to underlie the formation of neural representations (TallonBaudry et al., 1999). The present study is, however, the first to show that distinct percepts of physically identical stimuli are associated with distinct stimulus-locked $\beta$ - $/ \gamma$-band oscillations. This adds on to the list of functional similarities between stimulus-locked and nonstimulus-locked oscillations (TallonBaudry et al., 1999; Palva et al., 2002) and suggests that adequate neural representations of the present simple stimuli emerge already during the evoked oscillations and permit the early behavioral response (cf. Palva et al., 2002).

\section{Neural correlates of consciousness}

We found that neuronal activities at various stages of cortical processing both before and after the stimulus onset were correlated with perception. However, prestimulus activity, for instance, cannot be seen to underlie conscious perception per se. Moreover, even in the poststimulus time, it is likely that some neural processes are not causally related to and are not required by the conscious perception, despite being correlated with it. Those processes that suffice the minimal set of neural activities required by consciousness are commonly called the "neural correlates of consciousness" (NCCs) (Chalmers, 2002). Neuronal activities supporting conscious perception have been proposed to involve synchronous cell assemblies (Engel and Singer, 2001) and both bottom-up and top-down processing in a network spanning several levels of cortical hierarchy (Engel and Singer, 2001; Thompson and Varela, 2001; Crick and Koch, 2003). In our data, the $\alpha$-band stimulus locking was most selective to conscious perception, suggesting that it is an elementary part of the NCCs. Interestingly, at $\sim 100 \mathrm{~ms}$ from stimulus onset, the $\beta$-/ $\gamma$-band stimulus locking was observable simultaneously with emergent sensorifrontoparietal $\alpha$-band stimulus locking (Fig. 4C) (see supplemental video 2 , available at www.jneurosci.org as supplemen- 
tal material). This points to an intriguing possibility that, at $\sim 100$ $\mathrm{ms}$, the phase interactions of these oscillations underlie an NCC so that the $\alpha$-band component signals the attentional context and top-down selection of the $\beta$-/ $\gamma$-band components that signal the neural representation, i.e., the content of the percept (Palva et al., 2005).

\section{References}

Blankenburg F, Taskin B, Ruben J, Moosmann M, Ritter P, Curio G, Villringer A (2003) Imperceptible stimuli and sensory processing in humans. Science 299:1864.

Brazdil M, Rektor I, Daniel P, Dufek M, Jurak P (2001) Intracerebral eventrelated potentials to subtreshold target stimuli. Clin Neurophysiol 112:650-661.

Carlsson K, Petrovic P, Skare S, Petersson KM, Ingvar M (2000) Tickling expectations: neural processing in anticipation of a sensory stimulus. J Cogn Neurosci 12:691-703.

Castelo-Branco M, Neuenschwander S, Singer W (1998) Synchronization of visual responses between the cortex, lateral geniculate cortex, and retina in the anesthetized cat. J Neurosci 18:6395-6410.

Chalmers DJ (2002) What is the neural correlate of consciousness? In: Neural correlates of consciousness (Metzinger T, ed), pp 121-137. Cambridge, MA: MIT.

Colder BW, Tanenbaum L (1999) Dissociation of fMRI activation and awareness in auditory perception task. Brain Res Cogn Brain Res 8:177-184.

Coull JT (1998) Neural correlates of attention and arousal: insights from electrophysiology, functional neuroimaging and psychopharmacology. Prog Neurobiol 55:343-361.

Crick F, Koch C (2003) A framework for consciousness. Nat Neurosci 6:119-126.

Dehaene S, Naccache L, Cohen L, Bihan DL, Mangin JF, Poline JB, Riviere D (2001) Cerebral mechanisms of word masking and unconscious repetition priming. Nat Neurosci 4:752-758.

Engel AK, Singer W (2001) Temporal binding and the neural correlates of sensory awareness. Trends Cogn Sci 5:16-25.

Feinstein JS, Stein MB, Castillo GN, Paulus MP (2004) From sensory processes to conscious perception. Conscious Cogn 13:323-335.

Fries P, Roelfsema PR, Engel AK, Konig P, Singer W (1997) Synchronization of oscillatory responses in visual cortex correlates with interocular rivalry. Proc Natl Acad Sci USA 94:12699-12704.

Fries P, Neuenschwander S, Engel AK, Goebel R, Singer W (2001a) Rapid feature selective neuronal synchronization through correlated latency shifting. Nat Neurosci 4:194-200.

Fries P, Reynolds JH, Rorie AE, Desimone R (2001b) Modulation of oscillatory neuronal synchronization by selective visual attention. Science 29:1560-1563.

Fu KM, Foxe JJ, Murray MM, Higgins BA, Javitt DC, Schroeder CE (2001) Attention-depended suppression of distracter visual input can be crossmodally cued as indexed by anticipatory parieto-occipital alpha-band oscillations. Brain Res Cogn Brain Res 12:145-152.

Garcia-Larrea L, Bastuji H, Mauguiere F (1991) Mapping study of somatosensory evoked potentials during selective spatial attention. Electroencephalogr Clin Neurophysiol 80:201-214.

Halgren E, Boujon C, Clarke J, Wang C, Chauvel P (2002) Rapid distributed fronto-parieto-occipital processing stages during working memory in humans. Cereb Cortex 12:710-728.

Hari R, Karhu J, Hämäläinen M, Knuutila J, Salonen O, Sams M, Vilkman V (1993) Functional organization of the human first and second somatosensory cortices: a neuromagnetic study. Eur J Neurosci 5:724-734.

Jensen O, Gelfand J, Kounios J, Lisman JE (2002) Oscillations in the alpha band $(9-12 \mathrm{~Hz})$ increase with memory load during retention in a shortterm memory task. Cereb Cortex 12:877-882.

Jokeit H, Makeig S (1994) Different event-related patterns of gamma-band power in brain waves of fast- and slow-reacting subjects. Proc Natl Acad Sci USA 91:6339-6343.

Karhu J, Tesche CD (1999) Simultaneous early processing of sensory input in human primary (SI) and (SII) somatosensory cortices. J Neurophysiol 81:2017-2025.

Kastner S, Ungerleider LG (2000) Mechanisms of visual attention in the human cortex. Annu Rev Neurosci 23:315-341.
Kulics AT (1982) Cortical neural evoked correlates of somatosensory stimulus detection in the rhesus monkey. Electroencephalogr Clin Neurophysiol 53:78-93.

Lamme VAF (2003) Why visual attention and awareness are different. Trends Cogn Sci 7:12-18.

Libet B, Alberts WW, Wright Jr EW, Feinstein B (1967) Responses of human somatosensory cortex to stimuli below threshold for conscious sensation. Science 158:1597-1600.

Linkenkaer-Hansen K, Nikulin VV, Palva S, Ilmoniemi RJ, Palva JM (2004) Prestimulus oscillations enhance psychophysical performance in humans. J Neurosci 24:10186-10190.

Makeig S, Westerfield M, Jung TP, Covington J, Townsend J, Sejnowski TJ, Courchesne E (1999) Functionally independent components of the late positive event-related potential during visual spatial attention. J Neurosci 19:2665-2680.

Makeig S, Westerfield M, Jung TP, Enghoff S, Townsend J, Courchesne E, Sejnowski TJ (2002) Dynamic brain sources of visual evoked response. Science 295:690-694.

Makeig S, Debener S, Onton J, Delorme A (2004) Mining event-related brain dynamics. Trends Cogn Sci 8:204-210.

Marois R, Yi DJ, Chun MM (2004) The neural fate of consciously perceived and missed events during attentional blink. Neuron 41:465-472.

Mauguiere F, Merlet I, Forss N, Vanni S, Jousmaki V, Adeleine P, Hari R (1997) Activation of a distributed somatosensory cortical network in the human brain. A single dipole modeling study of magnetic fields evoked by median nerve stimulation. Part I: location and activation timing of SEF sources. Electroencephalogr Clin Neurophysiol 104:281-289.

Meador KJ, Ray PG, Echauz JR, Loring DW, Vachtsevanos GJ (2002) Gamma coherence and conscious perception. Neurology 59:847-854.

Naccache L, Dehaene S (2001) The priming method: imaging unconscious repetition priming reveals an abstract representation of number in the parietal lobes. Cereb Cortex 10:966-974.

Nikouline VV, Linkenkaer-Hansen K, Wikström H, Kesäniemi M, Antonova EV, Ilmoniemi RJ, Huttunen J (2000) Dynamics of mu-rhythm suppression caused by median nerve stimulation: a magnetoencephalographic study in human subjects. Neurosci Lett 24:163-166.

Palva JM, Palva S, Kaila K (2005) Phase synchrony among neuronal oscillations in the human cortex. J Neurosci 25:3962-3972.

Palva S, Palva JM, Shtyrov Y, Kujala T, Ilmoniemi RJ, Kaila K, Näätänen R (2002) Distinct gamma-band evoked responses to speech and nonspeech sounds in humans. J Neurosci 22:RC211(1-5).

Parasuraman R, Beatty J (1980) Brain events underlying detection and recognition of weak sensory signals. Science 210:80-83.

Penna SD, Torquati K, Pizzella V, Babiloni C, Franciotti R, Rossini PM, Romani GL (2004) Temporal dynamics of alpha and beta rhythms in human SI and SII after galvanic median nerve stimulation. NeuroImage 22:1438-1446.

Pfurtscheller G, Lopes da Silva FH (1999) Event-related EEG/MEG synchronization and desynchronization: basic principles. Clin Neurophysiol 110:1842-1857.

Pins D, Ffytche D (2003) The neural correlates of conscious vision. Cereb Cortex 13:461-474.

Ray PG, Meador KJ, Smith JR, Wheless JW, Sittenfeld M, Clifton GL (1999) Physiology of perception: cortical stimulation and recording in humans. Neurology 52:1044-1049.

Rees G, Kreiman G, Koch C (2002) Neural correlates of consciousness in humans. Nat Rev Neurosci 3:261-270.

Rodriguez E, George N, Lachaux JP, Martinerie J, Renault B, Varela FJ (1999) Perception's shadow: long-distance synchronization of human brain activity. Nature 397:430-433.

Sahraie A, Weiskrantz L, Barbur JL, Simmons A, Williams SC, Brammer MJ (1997) Pattern of neuronal activity associated with conscious and unconscious processing of visual signals. Proc Natl Acad Sci USA 94:9406-9411.

Simões C, Jensen O, Parkkonen L, Hari R (2003) Phase locking between human primary and secondary somatosensory cortices. Proc Natl Acad Sci USA 100:2691-2694.

Singer W (1999) Neuronal synchrony: a versatile code for the definition of relations? Neuron 24:49-65.

Singer W (2002) Consciousness from neurobiological perspective. In: Neural correlates of consciousness (Metzinger T, ed), pp 121-137. Cambridge, MA: MIT. 
Sinkkonen J, Tiitinen H, Näätänen R (1995) Gabor filters: an informative way for analysing event-related brain activity. J Neurosci Methods 56:99-104.

Srinivasan R, Russell DP, Edelman GM, Tononi G (1999) Increased synchronization of neuromagnetic responses during conscious perception. J Neurosci 19:5435-5448.

Steinmetz PN, Roy A, Fitzgerald PJ, Hsiao SS, Johnson KO, Niebur E (2000) Attention modulates synchronized neuronal firing in primate somatosensory cortex. Nature 404:187-190.

Supèr H, Spekreijse H, Lamme VAF (2001) Two distinct modes of sensory processing observed in monkey primary visual cortex (V1). Nat Neurosci 4:304-310.

Supèr H, van der Togt C, Spekreijse H, Lamme VAF (2003) Internal state of monkey primary visual cortex (V1) predicts figure-ground perception. J Neurosci 23:3407-3414.

Tallon-Baudry C, Bertrand O (1999) Oscillatory gamma activity in humans and its role in object representation. Trends Cogn Sci 3:151-162.

Thompson E, Varela FJ (2001) Radical embodiment: neural dynamics and consciousness. Trends Cogn Sci 5:418-425.
Tiitinen H, Sinkkonen J, Reinikainen K, Alho K, Lavikainen J, Näätänen R (1993) Selective attention enhances the auditory $40-\mathrm{Hz}$ transient response in humans. Nature 364:59-60.

Tononi G, Srinivasan R, Russell DP, Edelman GM (1998) Investigating neural correlates of conscious perception by frequency-tagged neuromagnetic responses. Proc Natl Acad Sci USA 203:3198-3203.

Van Rullen R, Koch C (2003) Is perception discrete or continuous? Trends Cogn Sci 7:207-213.

von Stein A, Chiang C, König P (2000) Top-down processing mediated by interareal synchronization. Proc Natl Acad Sci USA 97:14748-14753.

Wickens TD (2002) Elementary signal detection theory, Chap 1, pp 3-15. New York: Oxford UP.

Worden MS, Foxe JJ, Wang N, Simpson GW (2000) Anticipatory biasing of visuospatial attention indexed by retinotopically specific $\alpha$-band electroencephalography increases over occipital cortex. J Neurosci 20:RC63(1-6).

Yamagishi N, Callan DE, Goda N, Anderson SJ, Yoshida Y, Kawato M (2003) Attentional modulation of oscillatory activity in human visual cortex. NeuroImage 20:98-113.

Zeman A (2004) Theories of visual awareness. Prog Brain Res 144:321-329. 